\title{
新型龙胆酸衍生物的合成及其抑制酪氨酸酶活性研究
}

\author{
宋长伟 $a$ 熊丽丹 ${ }^{b}$ 王裕军 ${ }^{a}$ 张 南 $b$ \\ 李 霞 ${ }^{a}$ 李 颖 ${ }^{a}$ 李 利*,c 尹述凡*,a \\ $\left({ }^{a}\right.$ 四川大学化学学院 成都 610064) \\ $\left({ }^{b}\right.$ 四川省化妆品工程技术研究中心 成都 610064) \\ $\left({ }^{c}\right.$ 四川大学华西医院皮肤科 成都 610041)
}

\begin{abstract}
摘要 为发现新型的美白活性化合物, 以龙胆酸甲酯、卤代烃和 $\alpha$-羟基酸乙酯为原料, 合成了 10 个未见文献报道的 2 差基-5-烷 $\left(H\right.$ ) 氧基苯甲酸酯类衍生物( $\mathbf{3 a}, \mathbf{3 b}$ 和 $\mathbf{6} \mathbf{a} \sim \mathbf{6} \mathbf{h}$ ), 其结构经 ${ }^{1} \mathrm{H} N \mathrm{~N}$, IR, MS 和 HRMS 确认. 初步生物活性测试 结果表明 6a, 6b, 6e 和 $6 \mathrm{f}$ 具有较强的抑制酪氨酸酶活性, 进一步药理实验表明经取代改造后的 $6 \mathrm{a} \sim 6 \mathrm{~h}$, 其毒性和光学 毒性都相对龙胆酸甲酯和氢醌更低.
\end{abstract}

关键词＼cjkstart美白; 龙胆酸甲酯; 酪氨酸酶; 毒性; 光学毒性

\section{Synthesis and Inhibition of Tyrosinase Acitivity of New Gentisic Acid Derivatives}

\author{
Song, Changwei ${ }^{a} \quad$ Xiong, Lidan ${ }^{b} \quad$ Wang, Yujun ${ }^{a} \quad{\text { Zhang, } \text { Nan }^{b}}^{b}$ \\ $\mathrm{Li}, \mathrm{Xia}^{a} \quad \mathrm{Li}, \mathrm{Ying}^{a} \quad \mathrm{Li}, \mathrm{Li}^{*}, c \quad$ Yin, Shufan*,a \\ ( ${ }^{a}$ College of Chemistry, Sichuan University, Chengdu 610064) \\ ( ${ }^{b}$ Sichuan Province Cosmetics Engineering Technology Research Center, Chengdu 610064) \\ ( ${ }^{c}$ West China Hospital, Sichuan University, Dermatological Department, Chengdu 610041)
}

\begin{abstract}
To discover new whitening active compounds, ten novel 2-hydroxy-5-alkyl( $H)$ oxybenzoic ester derivatives (3a, 3b and $\mathbf{6 a} \sim \mathbf{6 h}$ ) were prepared via the starting materials of methyl gentisate, alkyl halides and $\alpha$-hydroxy acid ethyl ester. Their chemical structures were confirmed by ${ }^{1} \mathrm{H}$ NMR, IR, MS and HRMS techniques. The preliminary bioassay test demonstrated that compounds $\mathbf{6 a}, \mathbf{6 b}, \mathbf{6 e}$ and $\mathbf{6 f}$ had stronger inhibition of tyrosinase activity. Further pharmacological experiments showed that after substitution modification of compounds $\mathbf{6 a} \sim \mathbf{6 h}$, their toxicity and phototoxicity are lower than methyl gentisate and hydroquinone.
\end{abstract}

Keywords white; gentian methyl; tyrosinase; toxicity; optical toxicity

酪氨酸酶即多酚氧化酶，是二价铜离子与酶蛋白相 结合的一种金属酶, 广泛存在于动物、植物及真菌中 ${ }^{[1,2]}$. 它也是参与黑色素合成的关键酶之一, 控制着黑色素的 形成过程, 其活性程度对色素的沉积起主要作用 ${ }^{[3]}$. 目 前市场上广泛使用的许多美白祛斑产品都是通过抑制 酪氨酸酶途径发挥其作用. 包括龙胆酸衍生物、抗坏血. 酸衍生物、熊果苷和氢醌(Hydroquinone, HQ) 等. 但其中 许多化合物已经被证实对皮肤细胞有致变等毒性作 用 ${ }^{[4]}$. 因此, 继续寻找和发现新型、高效且安全的美白祛 斑活性化合物仍是当前研究的热点.
龙胆酸即 2,5-二羑基苯甲酸(gentisic acid，MA，2)， 是从龙胆根中提取的天然活性产物, 已在化妆品中作为 一种常规皮肤美白剂、抗氧化剂 ${ }^{[5]}$. 包括它的烷基酯类 衍生物也是一种很好的美白剂. 但它们对皮肤细胞的毒 副作用均较强，尽管其皮肤渗透率比较低本身也是其主 要缺陷之一 ${ }^{[6]}$. 鉴于此, 本工作以龙胆酸甲酯为先导物, 将可加速皮肤细胞角质层脱落并使表面肌肤光滑、柔细 的 $\alpha$-羟基酸等有效结构片段引入先导物中，设计并合成 了一系列 2-羊基-5-烷 $(H)$ 氧基苯甲酸酯类衍生物 3a, 3b, $\mathbf{6 a} \sim \mathbf{6 h}^{[7 \sim 9]}$, 期望通过有效分子片段的引入以增强其抑

*E-mail: chuandayouji217@163.com

Received January 7, 2012; revised March 14, 2012; published online May 11, 2012. 
制酪氨酸酶的活性，同时得到毒性更低、活性更强的美 白类化合物. 合成路线见 Scheme 1 .

\section{1 实验部分}

\section{1 仪器与试剂}

实验所用的试剂为分析纯或化学纯; 龙胆酸甲酯 (1)由北京中科扩展有限公司提供.

核磁共振(NMR): ${ }^{1} \mathrm{H}$ NMR 用 Bruker AVII-400 核磁 共振仪测定, 以 $\mathrm{CDCl}_{3}$ 为溶剂, TMS 作内标; 高分辨质 谱(HRMS): TOF-MS (Waters Q-TOF-Premiter instrument); 红外光谱(IR): Perkin-Elmer 16PC-FT (KBr 压片 或 $\mathrm{NaCl}$ 盐片); 熔点: Yanaco 显微熔点测定仪测定(温 度计未经校正). 电子天平: Sartorius BS210s; 薄层板; 硅胶 GF254(青岛海洋化工厂); 柱层析用 200 300 目试 剂级硅胶 (青岛海洋化工厂). ELISA 酶标仪 (BIO-RAD680 型).

氢醌(简称 $\mathrm{HQ}$ )：天津市美琳工贸有限公司, 纯度为 $98 \%$ 以上. 蘑菇酪氨酸酶(EC 1.14.1.8.1，30 U, Sigma, T3824).

\subsection{2-羟基-5-烷 $(H)$ 氧基苯甲酸酯类衍生物的合成}

\subsubsection{2-羟基-5-烷 $(H)$ 氧基苯甲酸 $(2$ 和 $\mathbf{5 a} \sim 5 \mathbf{d})$ 的合成}

取 $50 \mathrm{mg}(0.30 \mathrm{mmol})$ 的龙胆酸甲酯纯品, 溶于 10 $\mathrm{mL}$ 的 $95 \%$ 乙醇溶液, 并在室温搅拌下缓慢加入 $36 \mathrm{mg}$ $(0.90 \mathrm{mmol})$ 的 $\mathrm{NaOH}$ 固体, 然后升温至 $60{ }^{\circ} \mathrm{C}$ 搅拌反应, TLC 跟踪至水解完全. 水解完后用稀盐酸调节 $\mathrm{pH}$ 至 $2 \sim 3$. 将溶液浓缩至干得白色固体. 然后将白色固体烘
干后用无水乙醇溶解过滤，再将滤液减压浓缩至干得白 色固体 2, 同法可得 $\mathbf{5 a} \sim \mathbf{5 d}$.

1.2.2 2,5-二羟基苯甲酸烷氧基羰基酯 $(3 \mathbf{a}, 3 \mathbf{b})$ 的合 成

取 $100 \mathrm{mg}(0.65 \mathrm{mmol})$ 的 2 纯品, 溶于 $10 \mathrm{~mL}$ 无水 $\mathrm{CH}_{2} \mathrm{Cl}_{2}$ 溶液, 然后依次加入 $0.01 \mathrm{~g}(0.08 \mathrm{mmol})$ DMAP (4-二甲氨基吡啶), $0.11 \mathrm{~g}(1.1 \mathrm{mmol})$ 的乙醇酸乙酯, 室 温下搅拌反应 $5 \mathrm{~min}$ 后, 加入 $0.11 \mathrm{~g}(0.02 \mathrm{mmol}) \mathrm{DCC}$ (1,3-二环己基碳二亚胺), 继续室温搅拌反应, TLC 监测 反应，反应完成后，过滤，滤液减压浓缩，将粗产品经 柱层析 [淋洗液为 $V$ (乙酸乙酯 $): V($ 石油醚 $)=1: 8$ ]得白 色固体 3a，同法可得 3b.

2,5-二羟基苯甲酸乙氧基羰基甲酯(3a): 白色固体， 产率 50\%. m.p. $73 \sim 74{ }^{\circ} \mathrm{C} ;{ }^{1} \mathrm{H}$ NMR $\left(400 \mathrm{MHz}, \mathrm{CDCl}_{3}\right) \delta$ : $1.31\left(\mathrm{t}, J=7.2 \mathrm{~Hz}, 3 \mathrm{H}, \mathrm{CH}_{3}\right), 4.28(\mathrm{dd}, J=3.2,14.0 \mathrm{~Hz}$, $\left.2 \mathrm{H}, \mathrm{COOCH}_{2}\right), 4.85$ (s, 2H, $\left.\mathrm{COOCH}_{2} \mathrm{COO}\right), 6.88$ (d, $J=$ $8.8 \mathrm{~Hz}, 1 \mathrm{H}, \mathrm{ArH}), 7.04$ (dd, $J=3.2,8.8 \mathrm{~Hz}, 1 \mathrm{H}, \mathrm{ArH}), 7.35$ (d, $J=3.2 \mathrm{~Hz}, 1 \mathrm{H}, \mathrm{ArH}), 9.98$ (s, 1H, ArOH), 10.39 (s, 1H, ArOH); IR (KBr) v: 3419, 2977, 2922, 1728, 1688, 1629, 1487, 1487, 1429, 1398, 1322, 1291, 1191, 1094, 1047, $881,823,785,684 \mathrm{~cm}^{-1}$; HRMS (ESI) calcd for $\mathrm{C}_{11} \mathrm{H}_{12} \mathrm{O}_{6}$ $[\mathrm{M}+\mathrm{H}]^{+}$241.0707, found 241.0698; $[\mathrm{M}+\mathrm{Na}]^{+}$ 263.0526, found 263.0557; $[\mathrm{M}+\mathrm{K}]^{+}$279.0265, found 279.0309 .

2,5-二羟基苯甲酸(1-乙氧基羰基)乙酯(3b)：黄色固 体, 产率 45\%. m.p. $74 \sim 75{ }^{\circ} \mathrm{C}$; ${ }^{1} \mathrm{H}$ NMR (400 MHz, $\left.\mathrm{CDCl}_{3}\right) \delta: 1.31\left(\mathrm{t}, J=7.2 \mathrm{~Hz}, 3 \mathrm{H}, \mathrm{CH}_{3}\right), 1.62$ (d, $J=7.2$<smiles>COC(=O)c1cc(O)ccc1O</smiles>

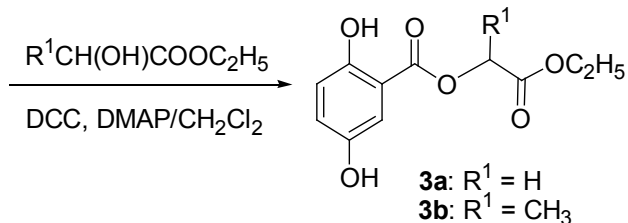

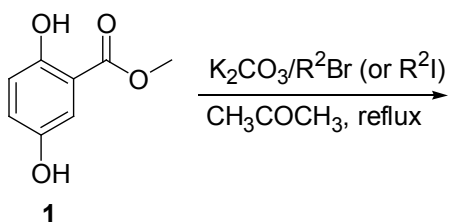<smiles>[Z10][Z10](=O)Oc1ccc(O)c(C(=O)OC)c1</smiles>

$\mathrm{NaOH}, \mathrm{MeOH} / \mathrm{H}_{2} \mathrm{O}$

$60{ }^{\circ} \mathrm{C}$<smiles>[R]Oc1ccc(O)c(C(=O)O[2H])c1</smiles>

5a $~ 5 d$. $90 \%$

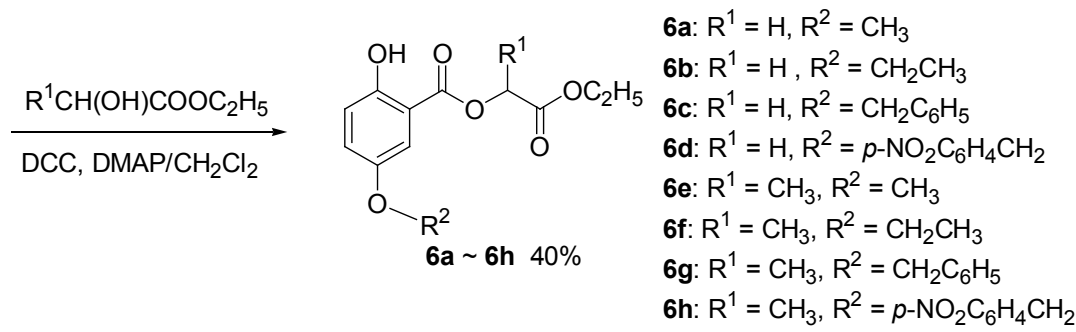

Scheme 1 
$\mathrm{Hz}, 3 \mathrm{H}, \mathrm{CH}_{3}$ ), 3.79 (s, 3H, $\mathrm{CH}_{3}$ ), 4.27 (dd, $J=3.2,14.4$ $\mathrm{Hz}, 2 \mathrm{H}, \mathrm{COOCH}_{2}$ ), 5.30 (dd, $J=3.2,14 \mathrm{~Hz}, 1 \mathrm{H}, \mathrm{COOCH}-$ COO), 6.82 (d, $J=8 \mathrm{~Hz}, 1 \mathrm{H}, \mathrm{ArH}$ ), 7.00 (dd, $J=3.2,8.8$ $\mathrm{Hz}, 1 \mathrm{H}, \mathrm{ArH}), 7.33$ (d, J=3.2 Hz, 1H, ArH), 10.03 (s, 1H, ArOH), 10.34 (s, 1H, ArOH); IR (KBr) v: 3402, 3248, 2991, 1727, 1678, 1629, 1467, 1401, 1290, 1227, 1198, $1096 \mathrm{~cm}^{-1}$; HRMS (ESI) calcd for $\mathrm{C}_{12} \mathrm{H}_{14} \mathrm{O}_{6}[\mathrm{M}+\mathrm{H}]^{+}$ 255.0863, found 255.0881; $[\mathrm{M}+\mathrm{Na}]^{+} 277.0683$, found 277.0703; [M+K $]^{+}$293.0422, found 293.0438.

\subsubsection{5-烷氧基-2-羟基苯甲酸甲酯 $4 \mathbf{a} \sim 4 \mathbf{d}$ 的合成}

取 $50 \mathrm{mg}(0.30 \mathrm{mmol})$ 的龙胆酸甲酯纯品, 溶于 10 $\mathrm{mL}$ 丙酮, 然后依次加入 $62 \mathrm{mg}(0.45 \mathrm{mmol})$ 的 $\mathrm{K}_{2} \mathrm{CO}_{3}$, $0.51 \mathrm{~g}(0.36 \mathrm{mmol})$ 的碘甲烷, 回流摚拌反应, TLC 跟踪 至反应完全, 过滤, 滤液减压浓缩, 将粗品经柱层析[淋 洗液为 $V($ 乙酸乙酯 $): V($ 石油醚 $)=1: 4]$ 分离纯化得 $\mathbf{4 a}$, 同法可得 $\mathbf{4 b} \sim \mathbf{4 d}$.

\subsubsection{2-羟基-5-烷氧基苯甲酸酯类 $6 \mathbf{a} \sim 6 \mathrm{~h}$ 的合成}

取 $100 \mathrm{mg}(0.55 \mathrm{mmol})$ 的 $\mathbf{5 a}$ 纯品, 溶于 $10 \mathrm{~mL}$ $\mathrm{CH}_{2} \mathrm{Cl}_{2}$ 溶液, 然后依次加入 $0.01 \mathrm{~g}(0.08 \mathrm{mmol})$ DMAP, $0.11 \mathrm{~g}(1.10 \mathrm{mmol})$ 的乙醇酸乙酯, 室温搅拌反应 $5 \mathrm{~min}$ 后, 加入 $0.11 \mathrm{~g}(0.02 \mathrm{mmol}) \mathrm{DCC}$, 继续室温摚拌反应, TLC 监测反应, 反应完全后, 过滤, 滤液减压浓缩, 将 粗产品经柱层析 [淋洗液为 $V$ (乙酸乙酯 $): V$ (石油醚 $)=$ $1: 8$ ]得白色固体 $\mathbf{6 a}$, 同法可得 $\mathbf{6 b} \sim 6 \mathbf{6 h}$.

2-羊基-5-甲氧基苯甲酸乙氧基羰基甲酯(6a): 白色 固体, 产率 70\%. m.p. $32 \sim 33{ }^{\circ} \mathrm{C} ;{ }^{1} \mathrm{H}$ NMR $(400 \mathrm{MHz}$, $\left.\mathrm{CDCl}_{3}\right) \delta: 1.31$ (t, $\left.J=7.2 \mathrm{~Hz}, 3 \mathrm{H}, \mathrm{CH}_{3}\right), 3.79$ (s, $3 \mathrm{H}, \mathrm{CH}_{3}$ ), 4.28 (dd, $\left.J=7.2,14.4 \mathrm{~Hz}, 2 \mathrm{H}, \mathrm{COOCH}_{2}\right), 4.87$ (s, $2 \mathrm{H}$, $\mathrm{COOCH}_{2} \mathrm{COO}$ ), $6.94(\mathrm{~d}, J=9.2 \mathrm{~Hz}, 1 \mathrm{H}, \mathrm{ArH}), 7.12$ (dd, $J=3.2,9.2 \mathrm{~Hz}, 1 \mathrm{H}, \mathrm{ArH}), 7.38(\mathrm{~d}, J=3.2 \mathrm{~Hz}, 1 \mathrm{H}, \mathrm{ArH})$, 10.05 (s, 1H, ArOH); IR (KBr) v: 3411, 2976, 2929, 2901, 1760, 1619, 1492, 1384, 1284, 1197, 1093, 1047, 880, 829, $786 \mathrm{~cm}^{-1}$; HRMS (ESI) calcd for $\mathrm{C}_{12} \mathrm{H}_{14} \mathrm{O}_{6}[\mathrm{M}+\mathrm{H}]^{+}$ 255.0863, found 255.0348; $[\mathrm{M}+\mathrm{Na}]^{+}$277.0683, found 277.0334; $[\mathrm{M}+\mathrm{K}]^{+}$293.0422, found 293.0105.

2-羟基-5-乙氧基苯甲酸乙氧基嶡基甲酯(6b): 白色 固体, 产率 68\%. m.p. 30 31 ${ }^{\circ} \mathrm{C} ;{ }^{1} \mathrm{H}$ NMR $(400 \mathrm{MHz}$, $\left.\mathrm{CDCl}_{3}\right) \delta: 1.31\left(\mathrm{t}, J=7.2 \mathrm{~Hz}, 3 \mathrm{H}, \mathrm{CH}_{3}\right), 1.40(\mathrm{t}, J=7.2 \mathrm{~Hz}$, $\left.3 \mathrm{H}, \mathrm{CH}_{3}\right), 1.65\left(\mathrm{~d}, J=7.2 \mathrm{~Hz}, 3 \mathrm{H}, \mathrm{CH}_{3}\right), 4.00$ (dd, $J=7.2$, $\left.14 \mathrm{~Hz}, 2 \mathrm{H}, \mathrm{OCH}_{2}\right), 4.28(\mathrm{dd}, J=7.2,14.4 \mathrm{~Hz}, 2 \mathrm{H}$, $\left.\mathrm{COOCH}_{2}\right), 4.86\left(\mathrm{~s}, 1 \mathrm{H}, \mathrm{COOCH}_{2} \mathrm{COO}\right), 6.93(\mathrm{~d}, J=8.8$ $\mathrm{Hz}, 1 \mathrm{H}, \mathrm{ArH}), 7.11$ (dd, $J=3.2,8.8 \mathrm{~Hz}, 1 \mathrm{H}, \mathrm{ArH}), 7.38(\mathrm{~d}$, $J=3.2 \mathrm{~Hz}, 1 \mathrm{H}, \mathrm{ArH}), 10.04$ (s, 1H, ArOH); IR (KBr) $v$ : 3251, 2982, 2937, 1762, 1687, 1618, 1492, 1426, 1385, 1326, 1286, 1191, 1094, 1035, 950, 831, $785 \mathrm{~cm}^{-1}$; HRMS
(ESI) calcd for $\mathrm{C}_{13} \mathrm{H}_{16} \mathrm{O}_{6}[\mathrm{M}+\mathrm{H}]^{+}$269.1020, found 269.1042; $[\mathrm{M}+\mathrm{Na}]^{+}$291.0839, found 291.08541; [M+ $\mathrm{K}]^{+}$307.0578, found 307.0621 .

2-羟基-5-苯甲氧基苯甲酸乙氧基羰基甲酯(6c): 白 色固体, 产率 65\%. m.p. $67 \sim 68{ }^{\circ} \mathrm{C} ;{ }^{1} \mathrm{H}$ NMR $(400 \mathrm{MHz}$, $\left.\mathrm{CDCl}_{3}\right) \delta: 1.31\left(\mathrm{t}, J=7.2 \mathrm{~Hz}, 3 \mathrm{H}, \mathrm{CH}_{3}\right), 4.28(\mathrm{dd}, J=7.2$, $\left.14 \mathrm{~Hz}, 2 \mathrm{H}, \mathrm{COOCH}_{2}\right), 4.86\left(\mathrm{~s}, 2 \mathrm{H}, \mathrm{OCH}_{2} \mathrm{Ph}\right), 5.03(\mathrm{~s}, 2 \mathrm{H}$, $\left.\mathrm{COOCH}_{2} \mathrm{COO}\right), 6.94(\mathrm{~d}, J=9.2 \mathrm{~Hz}, 1 \mathrm{H}, \mathrm{ArH}), 7.18(\mathrm{dd}$, $J=2.8,8.8 \mathrm{~Hz}, 1 \mathrm{H}, \mathrm{ArH}), 7.34 \sim 7.44$ (m, 5H, ArH), 7.49 (d, $J=2.8 \mathrm{~Hz}, 1 \mathrm{H}, \mathrm{ArH}), 10.07$ (s, 1H, ArOH); IR (KBr) $v$ : 3214, 2980, 2914, 2873, 1744, 1680, 1615, 1491, 1428, 1385, 1278, 1240, 1194, 1090, 1017, 840, 787, 750, 701 $\mathrm{cm}^{-1}$; HRMS (ESI) calcd for $\mathrm{C}_{18} \mathrm{H}_{18} \mathrm{O}_{6}[\mathrm{M}+\mathrm{H}]^{+}$ 331.1176, found 331.1173; $[\mathrm{M}+\mathrm{Na}]^{+}$353.0996, found 353.0996; $[\mathrm{M}+\mathrm{K}]^{+}$369.0735, found 369.0717.

2-差基-5-(4-硝基苯甲氧基)苯甲酸乙氧基羰基甲酯 (6d): 白色固体, 产率 64\%. m.p. 67 68 ${ }^{\circ} \mathrm{C} ;{ }^{1} \mathrm{H}$ NMR (400 MHz, $\left.\mathrm{CDCl}_{3}\right) \delta: 1.32\left(\mathrm{t}, J=7.2 \mathrm{~Hz}, 3 \mathrm{H}, \mathrm{CH}_{3}\right), 1.65$ (d, $\left.J=7.2 \mathrm{~Hz}, 3 \mathrm{H}, \mathrm{CH}_{3}\right), 4.28(\mathrm{dd}, J=7.2,14 \mathrm{~Hz}, 2 \mathrm{H}$, $\left.\mathrm{COOCH}_{2}\right), 4.86\left(\mathrm{~s}, 2 \mathrm{H}, \mathrm{OCH}_{2} \mathrm{PhNO}_{2}\right), 5.14(\mathrm{~s}, 2 \mathrm{H}$, $\mathrm{COOCH}_{2} \mathrm{COO}$ ), 6.97 (d, J=9.2 Hz, 1H, ArH), 7.19 (dd, $J=3.2,9.2 \mathrm{~Hz}, 1 \mathrm{H}, \mathrm{ArH}), 7.47(\mathrm{~d}, J=3.2 \mathrm{~Hz}, 1 \mathrm{H}, \mathrm{ArH})$, $7.61\left(\mathrm{~d}, J=9.2 \mathrm{~Hz}, 2 \mathrm{H}, \mathrm{ArNO}_{2}\right), 8.26(\mathrm{~d}, J=8.8 \mathrm{~Hz}, 2 \mathrm{H}$, $\mathrm{ArNO}_{2}$ ), 10.09 (s, 1H, ArOH); IR (KBr) v: 3125, 2919, 2850, 2342, 1746, 1685, 1541, 1473, 1420, 1397, 1341, $1235,1189,1094,1030,855,829,743 \mathrm{~cm}^{-1}$; HRMS (ESI) calcd for $\mathrm{C}_{19} \mathrm{H}_{19} \mathrm{NO}_{8}[\mathrm{M}+\mathrm{H}]^{+}$376.1027, found 376.1036; $[\mathrm{M}+\mathrm{Na}]^{+}$398.0846, found 398.0843; [M+K] 414.0586, found 414.0539.

2-着基-5-甲氧基苯甲酸(1-乙氧基羰基)乙酯(6e): 白色固体, 产率 $48 \%$. m.p. $34 \sim 36{ }^{\circ} \mathrm{C} ;{ }^{1} \mathrm{H}$ NMR (400 $\left.\mathrm{MHz}, \mathrm{CDCl}_{3}\right) \delta: 1.30$ (t, $\left.J=7.2 \mathrm{~Hz}, 3 \mathrm{H}, \mathrm{CH}_{3}\right), 1.66(\mathrm{~d}, J=$ $\left.7.2 \mathrm{~Hz}, 3 \mathrm{H}, \mathrm{CH}_{3}\right), 3.79$ (s, $\left.3 \mathrm{H}, \mathrm{CH}_{3}\right), 4.25$ (dd, $J=7.2,14.0$ $\left.\mathrm{Hz}, 2 \mathrm{H}, \mathrm{COOCH}_{2}\right), 5.32(\mathrm{dd}, J=7.2,14.0 \mathrm{~Hz}, 1 \mathrm{H}$, COOCHCOO), 6.93 (d, $J=9.2 \mathrm{~Hz}, 1 \mathrm{H}, \mathrm{ArH}), 7.11$ (dd, $J=3.2,9.2 \mathrm{~Hz}, 1 \mathrm{H}, \mathrm{ArH}), 7.37(\mathrm{~d}, J=3.2 \mathrm{~Hz}, 1 \mathrm{H}, \mathrm{ArH})$, 10.12 (s, 1H, ArOH); IR (KBr) v: 3261, 2977, 2939, 2835, 1751, 1683, 1618, 1493, 1456, 1385, 1366, 1284, 1229, $1098,1038,849,782,718 \mathrm{~cm}^{-1}$; HRMS (ESI) calcd for $\mathrm{C}_{13} \mathrm{H}_{16} \mathrm{O}_{6}[\mathrm{M}+\mathrm{H}]^{+}$269.1020, found 269.1078; [M+Na] $]^{+}$ 291.0839, found 291.0839; $[\mathrm{M}+\mathrm{K}]^{+}$307.0578, found 307.0607 .

2-羊基-5-乙氧基苯甲酸(1-乙氧基羰基)乙酯(6f): 浅 黄色固体, 产率 $65 \%$. m.p. $72 \sim 73{ }^{\circ} \mathrm{C} ;{ }^{1} \mathrm{H}$ NMR (400 $\left.\mathrm{MHz}, \mathrm{CDCl}_{3}\right) \delta: 1.32\left(\mathrm{t}, J=7.2 \mathrm{~Hz}, 3 \mathrm{H}, \mathrm{CH}_{3}\right), 1.65(\mathrm{~d}, J=$ $\left.7.2 \mathrm{~Hz}, 3 \mathrm{H}, \mathrm{CH}_{3}\right), 3.98 \sim 4.04\left(\mathrm{~m}, 2 \mathrm{H}, \mathrm{OCH}_{2}\right), 4.25(\mathrm{dd}$, 
$\left.J=7.2,14.4 \mathrm{~Hz}, 2 \mathrm{H}, \mathrm{COOCH}_{2}\right), 5.31(\mathrm{dd}, J=6.8,14.0 \mathrm{~Hz}$, $1 \mathrm{H}, \mathrm{COOCHCOO}), 6.922(\mathrm{~d}, J=8.8 \mathrm{~Hz}, 1 \mathrm{H}, \mathrm{ArH}), 7.10$ (dd, $J=3.2,8.8 \mathrm{~Hz}, 1 \mathrm{H}, \mathrm{ArH}), 7.38(\mathrm{~d}, J=3.2 \mathrm{~Hz}, 1 \mathrm{H}$, ArH), 10.09 (s, 1H, ArOH); IR (KBr) v: 3261, 2983, 2943, 2877, 1751, 1682, 1617, 1495, 1367, 1286, 1229, 1182, 1098, 1043, 854, 788, $726 \mathrm{~cm}^{-1}$; HRMS (ESI) calcd for $\mathrm{C}_{14} \mathrm{H}_{18} \mathrm{O}_{6}[\mathrm{M}+\mathrm{H}]^{+}$283.1176, found 283.1185; [M+Na $]^{+}$ 305.0996, found 305.1020; $[\mathrm{M}+\mathrm{K}]^{+}$321.0735, found 321.0748 .

2-羟基-5-苯甲氧基苯甲酸(1-乙氧基羰基)乙酯( $6 \mathbf{g}$ ): 白色固体, 产率 64\%. m.p. 31 32 ${ }^{\circ} \mathrm{C} ;{ }^{1} \mathrm{H}$ NMR (400 $\left.\mathrm{MHz}, \mathrm{CDCl}_{3}\right) \delta: 1.30\left(\mathrm{t}, J=7.2 \mathrm{~Hz}, 3 \mathrm{H}, \mathrm{CH}_{3}\right), 1.65$ (d, $J=$ $\left.7.2 \mathrm{~Hz}, 3 \mathrm{H}, \mathrm{CH}_{3}\right), 3.79\left(\mathrm{~s}, 3 \mathrm{H}, \mathrm{CH}_{3}\right), 4.25$ (dd, $J=7.2,14.4$ $\left.\mathrm{Hz}, 2 \mathrm{H}, \mathrm{COOCH}_{2}\right), 5.03$ (s, $2 \mathrm{H}, \mathrm{OCH}_{2} \mathrm{Ph}$ ), 5.31 (dd, $J=$ 6.8, $14.0 \mathrm{~Hz}, 1 \mathrm{H}, \mathrm{COOCHCOO}), 6.93(\mathrm{~d}, J=9.2 \mathrm{~Hz}, 1 \mathrm{H}$, ArH), 7.17 (dd, $J=3.2,9.2 \mathrm{~Hz}, 1 \mathrm{H}, \mathrm{ArH}), 7.34 \sim 7.45$ (m, 5H, ArH), 7.48 (d, $J=3.2 \mathrm{~Hz}, 1 \mathrm{H}, \operatorname{ArH}), 10.12(\mathrm{~s}, 1 \mathrm{H}$, ArOH); IR (KBr) v: 3223, 2986, 2940, 1753, 1681, 1615, 1489, 1454, 1380, 1281, 1199, 1097, 1019, 860, 786, 749, $699 \mathrm{~cm}^{-1}$; HRMS (ESI) calcd for $\mathrm{C}_{19} \mathrm{H}_{20} \mathrm{O}_{6}[\mathrm{M}+\mathrm{H}]^{+}$ 345.1333, found 345.1330; $[\mathrm{M}+\mathrm{Na}]^{+} 367.1152$, found 367.1145; $[\mathrm{M}+\mathrm{K}]^{+}$383.0891, found 383.0887.

2-羟基-5-(4-硝基苯甲氧基)苯甲酸(1-乙氧基羰基) 乙酯(6h): 浅黄色固体, 产率 $60 \%$. m.p. $55 \sim 56{ }^{\circ} \mathrm{C} ;{ }^{1} \mathrm{H}$ NMR (400 MHz, $\left.\mathrm{CDCl}_{3}\right) \delta: 1.30$ (t, $J=7.2 \mathrm{~Hz}, 3 \mathrm{H}, \mathrm{CH}_{3}$ ), $1.65\left(\mathrm{~d}, J=7.2 \mathrm{~Hz}, 3 \mathrm{H}, \mathrm{CH}_{3}\right), 4.26(\mathrm{dd}, J=7.2,14.0 \mathrm{~Hz}$, $2 \mathrm{H}, \mathrm{COOCH}_{2}$ ), 5.15 (s, $2 \mathrm{H}, \mathrm{OCH}_{2} \mathrm{PhNO}_{2}$ ), 5.32 (dd, $J=$ 7.2, $14.0 \mathrm{~Hz}, 1 \mathrm{H}, \mathrm{COOCHCOO}), 6.95(\mathrm{~d}, J=9.2 \mathrm{~Hz}, 1 \mathrm{H}$, ArH), 7.17 (dd, $J=3.2,9.2 \mathrm{~Hz}, 1 \mathrm{H}, \mathrm{ArH}), 7.46$ (d, $J=3.2$ $\mathrm{Hz}, 1 \mathrm{H}, \mathrm{ArH}), 7.63\left(\mathrm{~d}, J=8.8 \mathrm{~Hz}, 2 \mathrm{H}, \mathrm{ArNO}_{2}\right), 8.26(\mathrm{~d}$, $\left.J=8.8 \mathrm{~Hz}, 2 \mathrm{H}, \mathrm{ArNO}_{2}\right), 10.15$ (s, 1H, ArOH); IR (KBr) $v$ : 3206, 2926, 1745, 1682, 1620, 1522, 1490, 1403, 1341, 1288, 1198, 1108, 1030, 855, 828, 789, 743, $701 \mathrm{~cm}^{-1}$; HRMS (ESI) calcd for $\mathrm{C}_{19} \mathrm{H}_{19} \mathrm{NO}_{8}[\mathrm{M}+\mathrm{H}]^{+} 390.1183$, found 390.1155; $[\mathrm{M}+\mathrm{Na}]^{+}$412.1003, found 412.0984; $[\mathrm{M}+\mathrm{K}]^{+}$428.0742, found 428.0727 .

\section{3 酪氨酸酶活性抑制实验}

采用蘑菇酪氨酸酶多巴速率氧化法, 以酶联免疫检 测仪于 $490 \mathrm{~nm}$ 处检测反应产物特异性吸光度 $\left(A_{490}\right)$ 值. 将化合物 $\mathbf{1}, \mathbf{3 a}, \mathbf{6 a} \sim \mathbf{6} \mathbf{h}$ 和 HQ 用 DMSO(二甲基亚砜)溶 解后, 用 $0.067 \mathrm{~mol} / \mathrm{L}, \mathrm{pH} 6.8$ 磷酸缓冲稀释配制成 6 个 浓度的溶液. 反应在 96 孔培养板中进行, 总反应体系 $200 \mu \mathrm{L}: 0.15 \%$ 的 $L$-Dopa $50 \mu \mathrm{L} ; 50 \mathrm{U} / \mathrm{mL}$ 酪氨酸酶 50 $\mu \mathrm{L}$; 测试药物 $50 \mu \mathrm{L} ; 0.067 \mathrm{~mol} / \mathrm{L}, \mathrm{pH} 6.8$ 磷酸缓冲液 50 $\mu \mathrm{L}$. 加样完毕, 将 96 孔培养板置于 $37{ }^{\circ} \mathrm{C}$ 孵育 $10 \mathrm{~min}$, 在酶标仪下测定每孔 $A_{490}$ 值. 最后计算酪氨酸酶的抑制
率, 酪氨酸酶抑制率 $=[(A-B)-(C-D)] /(A-$ $B) \times 100 \% . A$ : 未加中药样品的加酶混合液所测的吸光 度; $B$ : 未加中药样品亦未加酶的混合液所测的吸光度; $C$ : 加中药样品和酶的混合液所测的吸光度; $D$ : 加中药 样品而未加酶的混合液所测的吸光度. 每次实验重复测 试 3 次.

\subsection{MTT 法检测化合物对细胞毒性}

细胞毒性实验参考文献[10]进行. 培养 3T3 细胞, 收集对数期细胞, 调整细胞悬液浓度 (由于 $3 \mathrm{~T} 3$ 细胞是 半贴壁细胞, 不用肤酶消化), 收集细胞, 重悬于 $\operatorname{DMEM}$ (低糖, 含 $4.0 \mathrm{mmol} \cdot \mathrm{L}^{-1} \mathrm{~L}$-谷氨酰胺、 $110 \mathrm{mg}$ 丙 酩酸钠)培养液, 配成 $3 \times 10^{4}$ 个细胞 $/ \mathrm{mL}$ 的细胞悬液, 共 $10 \mathrm{~mL} .96$ 孔板中每孔加入 $100 \mu \mathrm{L}$ 细胞悬液, 置于 $37^{\circ} \mathrm{C}$ 孵箱中培养 $24 \mathrm{~h}$. 将配制好的浓度为 $2 \mathrm{mg} / \mathrm{mL}$ 的龙胆酸 衍生物 $3 \mathbf{a}, \mathbf{6 a} \sim 6 \mathbf{h}$ 和氢醌(1) 在超净工作台中, 用 0.22 $\mu \mathrm{m}$ 的过滤器过滤水, 去除细菌. 以 DMEM 为稀释液, 制备不同浓度梯度的龙胆酸衍生物 $3 \mathrm{a}, \mathbf{6 a} \sim 6 \mathrm{~h}$ 和 1 . 按 照浓度梯度从低到高 $(0.25 \sim 250 \mu \mathrm{g} / \mathrm{mL})$ 依次在每行中 分别加入同一稀释度的 $100 \mu \mathrm{L}$, 每个浓度各做 5 个复孔. 并用一列作为空白对照，即只用加入 $100 \mu \mathrm{L}$ 的 DMEM 双有培养液[含 $10 \% \mathrm{FBS}$ (胎牛血清)、 $1 \%$ 双抗]. 置于 37 ${ }^{\circ} \mathrm{C}$ 捊箱中培养 $24 \mathrm{~h}$. 倒置显微镜下观察. 在每孔中加入 浓度为 $5 \mathrm{mg} / \mathrm{mL}$ MTT [3-(4,5-二甲基噻唑-2)-2,5-二苯基 四氮唑溴盐]液 $20 \mu \mathrm{L}$, 置于 $37^{\circ} \mathrm{C}$ 孵箱中培养 $3 \sim 4 \mathrm{~h}$. 吸 出孔内培养液后(若药物与 MTT 能够反应, 可先弃去培 养液, 用 PBS 冲 2 3 遍后, 再加入含 MTT 的培养液.) 每孔加入 $150 \mu \mathrm{L}$ DMSO, 将 96 孔板置于微孔板振荡器 上震荡 $5 \mathrm{~min}$, 使蓝色结晶物溶解. 用酶标仪在 $570 \mathrm{~nm}$ 下检测各孔的 $\mathrm{OD}$ 值. $\mathrm{RGR}=($ 实验组平均 $\mathrm{OD}$ 值/空白对 照组平均 OD 值) $\times 100 \%$, 转化为相应的细胞毒性等级, 标准为: 0 级为 $R G R \geqslant 100 \% ; 1$ 级为 $75 \% \sim 99 \%$; 2 级为 $50 \% \sim 74 \%$; 级为 $25 \% \sim 49 \%$; 级为 $1 \% \sim 24 \%$; 增殖 率 $\leqslant 0$ 者为 5 级.

\section{5 光毒性实验}

光毒性实验参考文献[11]进行. 收集处于对数生长 期的 $3 \mathrm{~T} 3$ 细胞, 细胞计数后, 将 $3 \mathrm{~T} 3$ 细胞转移到 96 孔板 中, 培养基调节细胞浓度, 保证每个孔中含有大约 $1 \times 10^{4}$ 个细胞. 将 96 孔板放置在 $37{ }^{\circ} \mathrm{C}, 5 \% \mathrm{CO}_{2}$ 的培养 箱中培养 $24 \mathrm{~h}$. 吸出培养液, 在每个孔中加入 $150 \mu \mathrm{L}$ 的 PBS 溶液洗两次. 分别用 8 种不同浓度, $100 \mu \mathrm{L}$ 的受试 物处理细胞(每个浓度作 6 个孔); 受试物先溶解于易溶 的溶剂中，再用培养基稀释到指定浓度. 将 96 孔板放置 在 $37{ }^{\circ} \mathrm{C}, 5 \% \mathrm{CO}_{2}$ 的培养箱中培养 $1 \mathrm{~h}$. 阳性组室温下曝 露在 UVA $\left(1.7 \mathrm{~mW} / \mathrm{cm}^{2}\right.$, 即 $\left.5 \mathrm{~J} / \mathrm{cm}^{2}\right)$ 下 $50 \mathrm{~min}$ 以评价光 毒性; 阴性组室温下放置于黑暗环境中 $50 \mathrm{~min}$ (如果条 
件允许, 最好用风扇吹动空气避免 96 孔板上形成水珠). 吸出受试液, 然后在每个孔中加入 $150 \mu \mathrm{L}$ 的 PBS 洗两 次. 在每个孔中加入 $100 \mu \mathrm{L}$ 培养基, 将 96 孔板放置在 $37{ }^{\circ} \mathrm{C}, 5 \% \mathrm{CO}_{2}$ 的培养箱中培养 $18 \sim 22 \mathrm{~h}$. 取出 96 孔板, 在显微镜下观察细胞形态学变化 (主要是细胞大小, 形 状和大概的贴壁细胞数量). 吸出培养基, 在每个孔中 加入 $150 \mu \mathrm{L}$ 的中性红培养液 $(50 \mu \mathrm{g} / \mathrm{mL})$; 将 96 孔板放置 在 $37{ }^{\circ} \mathrm{C}, 5 \% \mathrm{CO}_{2}$ 的培养箱中培养 $3 \mathrm{~h}$. 吸出中性红培养 液, 在每个孔中加入 $150 \mu \mathrm{L}$ 的 PBS 洗一次; 再在每个孔 中加入 $150 \mu \mathrm{L}$ 的解吸附液 $\left(V_{\text {水 }}: V_{\text {乙醇 }}: V_{\text {冰酷酸 }}=\right.$ $49: 50: 1)$. 室温下震荡平板 $10 \mathrm{~min}$. 在 $540 \mathrm{~nm}$ 波长下 读取吸光度, 检测细胞对中性红的吸收情况 (反映细胞 活性). 确定以光刺激因子(PIF 值)为基础的预测模型: 通过分析有光照 $(\mathrm{UVA}+)$ 和无光照 $(\mathrm{UVA}-)$ 两种情况下 获得的细胞毒性浓度反应曲线, 确定能抑制 $50 \%$ 细胞活 性的受试物浓度 $\left(\mathrm{EC}_{50}\right)$ 来计算光刺激因子 $(\mathrm{PIF}): \mathrm{PIF}=$ $\mathrm{EC}_{50}(\mathrm{UVA}-) / \mathrm{EC}_{50}(\mathrm{UVA}+)$. 评价标准为: 如果 $\mathrm{PIF}<2$ : 预测无潜在光毒性; 如果 $\mathrm{PIF}>5$ : 预测有潜在光毒性; 如果 PIF 在 2 5 之间, 需要重新进行证实实验再判断. 受试物在 UV+下有细胞毒性, UV一条件下无细胞毒性 时, 如果阴性组所使用的浓度已经达到最高受试浓度 $\left(C_{\max }\right)$, 可计算 $>\operatorname{PIF}$ (如果只获得一个 $>\mathrm{PIF}$, 那么任何 $>\mathrm{PIF}>1$ 的值都能提示有潜在的光毒性: $>\mathrm{PIF}=$ $C_{\max }(\mathrm{UV}-) / \mathrm{EC}_{50}(\mathrm{UV}+)$.

\section{2 结果与讨论}

\section{1 合成}

根据文献[12]合成了 $\mathbf{4 a \sim 4 d}$. 实验发现, 用丙酮作 溶剂时, 只得到 5-位着基上的烷基化产物, 主要原因可 能是一方面 2 位羟基旁位阻较大的原因, 另一方面 2 位 羟基容易与羰键形成分子内氢键导致 2-位羟基上的烷 基化反应相当困难. 当碳酸钾和龙胆酸甲酯、卤代烷按 物质的量比 $1: 1: 1$ 投料, 龙胆酸甲酯反应不完全; 增 大卤代烃的用量产率提高也不明显, 而增大碳酸钾的用 量, 可增加龙胆酸甲酯的反应产率; 实验结果表明当碳 酸钾和龙胆酸甲酯、卤代烃投料比为 $1.5: 1: 1.2$ 时, 可 获得较高的产率. 在化合物 $\mathbf{5 a} \sim \mathbf{5 d}$ 的合成过程中, 水解 温度在 $60 \sim 65{ }^{\circ} \mathrm{C}$ 时, 原料可以彻底水解, 达到一个很 高的水解产率. 参考文献[13], 用 DCC-DMAP 方法合成 了 $6 \mathrm{a} \sim 6 \mathrm{~d}$. 在合成过程中, 发现如果将 DCC 的同时投 料改为在酸、醇和 DMAP 投完摚拌一段时间后再加入, 可以获得较高的产率, 而且副产物的量也很少.

\section{2 波谱分析}

\subsection{1 ${ }^{1} \mathrm{H}$ NMR 谱图}

所合成的化合物 $3 \mathbf{a}, \mathbf{3 b}$, 苯环上 2 位上的酚羟基质
子化学位移都出现在 $\delta 9.98 \sim 10.15$ 之间, 5 位上的酚羟 基质子化学位移出现在了 $\delta \quad 10.34 \sim 10.39$ 之间. 在 $\delta$ 6.81 7.46 范围内均有苯环上 3 个氢的化学位移. 而所 合成的化合物 $\mathbf{6 a} \sim \mathbf{6 h}$, 化学位移在 $\delta 10.34 \sim 10.39$ 之间 均已消失，提示 5 位上的羟基已被取代，而化学位移在 $\delta 9.98 \sim 10.15$ 之间仍然保持了苯环 2 位上的酚羟基质子 化学位移.

\subsubsection{IR 谱图}

所合成的化合物 3a, 3b, 6a $\sim 6 \mathbf{h}$ 中, 3420 或 $3200 \mathrm{~cm}^{-1}$ 附近出现宽而强的 $\mathrm{O}-\mathrm{H}$ 伸缩振动吸收峰; $2990 \mathrm{~cm}^{-1}$ 左 右区域系甲基、亚甲基、次甲基碳氢键伸缩振动吸收峰; 1620 1750 $\mathrm{cm}^{-1}$ 范围内出现酯基中 $\mathrm{C}=\mathrm{O}$ 的吸收峰; $1600 \sim 1450 \mathrm{~cm}^{-1}$ 为苯环的骨架伸缩振动峰; $1275 \sim 650$ $\mathrm{cm}^{-1}$ 也出现了苯环上 $\mathrm{C}-\mathrm{H}$ 变形振动的吸收峰.

\subsubsection{HRMS 谱图}

目标化合物 3a, 3b, 6a $\sim 6 h$ 质子峰都以准分子离子 峰 $[\mathrm{M}+\mathrm{H}]^{+}$或 $[\mathrm{M}+\mathrm{Na}]^{+},[\mathrm{M}+\mathrm{K}]^{+}$基峰的形式出现, 说 明化合物均较稳定. HRMS 检测结果与目标化合物的分 子组成一致.

\section{3 生物活性实验}

\subsection{1 酪氨酸酶活性抑制实验}

酪氨酸酶活性抑制实验结果如表 1 所示. 从表 1 的 数据可以看出 $6 \mathrm{a}, 6 \mathrm{~b}, 6 \mathrm{e}, 6 \mathrm{f}$ 表现出比较明显的抑制酪氨 酸酶活性作用. 而 3a, 6c, 6d, 6g, 6h 虽然抑制酪氨酸酶 活性作用不够显著, 可能是其溶解度低导致的晶体析出 现象, 但实验中发现其可能会与多巴反应.

\subsubsection{MTT 法检测化合物对细胞毒性}

MTT 法检测化合物对细胞毒性实验结果如表 2 所 示. 从表 2 的数据中可以看出未经过取代保护的 $\mathbf{3 a}$ 与原 料药 1 和氢醌毒性相比, 变化不大, 都具有较强的细胞 毒性, 而经过 5 位取代保护的 $6 \mathbf{a} \sim \mathbf{6 h}$ 可以看出毒性测定 结果明显较低, 尤其是化合物 $\mathbf{6 b} \sim \mathbf{6 d}, \mathbf{6 f}, \mathbf{6 g}$ 可以看出 具有很低的药物毒性. 从目标化合物的结构上可以看出 把苯环 5 位上的羟基进行取代保护后可以明显降低目标 化合物的细胞毒性.

\subsection{3 光毒性实验}

各化合物对细胞光毒性实验测定结果如表 3 所示. 从表 3 的数据中可以看出, 化合物 $6 \mathrm{~b}, 6 \mathrm{e} \sim 6 \mathrm{~g}$ 具有较低 的光学毒性, 化合物 $6 \mathrm{a}, 6 \mathrm{c}, 6 \mathrm{~d}, 6 \mathrm{~h}$ 无潜在的光学毒性. 从目标化合物结构可以看出同样将苯环 5 位羟基进行取 代保护可以明显减小目标化合物的光毒性，而未经保护 的化合物 3a 则具有较强的光毒性, 但它们均较氢醌与 先导物龙胆酸甲酯的毒性明显要低. 进一步的药理毒理 研究仍在进行中. 
表 1 目标化合物不同浓度对酪氨酸酶活性抑制百分率的测定结果

Table 1 The measured results of different concentrations of target compounds on tyrosinase activity inhibition percentage

\begin{tabular}{|c|c|c|c|c|c|c|}
\hline 化合物 & $4 \mathrm{mg} / \mathrm{mL}$ & $2 \mathrm{mg} / \mathrm{mL}$ & $1 \mathrm{mg} / \mathrm{mL}$ & $500 \mu \mathrm{g} / \mathrm{mL}$ & $250 \mu \mathrm{g} / \mathrm{mL}$ & $62.5 \mu \mathrm{g} / \mathrm{mL}$ \\
\hline HQ & $31.51 \pm 0.90$ & $12.32 \pm 1.03$ & - & - & - & - \\
\hline 1 & $20.85 \pm 0.40$ & $17.06 \pm 0.59$ & $6.16 \pm 0.66$ & - & - & - \\
\hline $3 \mathbf{a}$ & $17.06 \pm 1.78$ & $11.37 \pm 0.76$ & - & - & - & - \\
\hline $6 \mathbf{a}$ & $4.98 \pm 0.28$ & $36.01 \pm 1.73$ & $33.65 \pm 1.21$ & $21.56 \pm 0.87$ & $15.88 \pm 0.67$ & $13.74 \pm 0.64$ \\
\hline $6 b$ & - & - & $28.67 \pm 0.32$ & $40.52 \pm 0.56$ & $25.82 \pm 0.22$ & $14.45 \pm 0.18$ \\
\hline $6 c$ & - & - & $18.00 \pm 0.13$ & $10.18 \pm 0.08$ & $11.13 \pm 0.09$ & $8.77 \pm 0.09$ \\
\hline 6d & - & $1.89 \pm 0.06$ & $7.81 \pm 0.16$ & $9.24 \pm 0.13$ & $9.95 \pm 0.19$ & $11.61 \pm 0.24$ \\
\hline $6 e$ & $60.43 \pm 1.89$ & $49.05 \pm 1.36$ & $36.02 \pm 1.41$ & $28.43 \pm 0.96$ & $23.45 \pm 1.24$ & $19.19 \pm 0.63$ \\
\hline $6 f$ & - & - & $27.25 \pm 0.43$ & $25.59 \pm 0.07$ & $11.61 \pm 0.04$ & $4.50 \pm 0.03$ \\
\hline $6 g$ & - & $27.25 \pm 0.86$ & $23.93 \pm 0.74$ & $7.58 \pm 0.61$ & $7.34 \pm 0.12$ & $0.23 \pm 0.05$ \\
\hline $6 \mathrm{~h}$ & $5.92 \pm 0.33$ & $21.56 \pm 0.84$ & $18.89 \pm 0.61$ & $12.80 \pm 0.26$ & $12.08 \pm 0.16$ & $9.00 \pm 0.15$ \\
\hline
\end{tabular}

表 2 目标化合物毒性的测定结果

Table 2 The results of target compounds of toxicity determination

\begin{tabular}{|c|c|c|c|c|c|c|c|c|c|c|c|c|}
\hline \multirow{2}{*}{$\begin{array}{l}\text { 化合 } \\
\text { 物 }\end{array}$} & \multicolumn{2}{|c|}{$250 \mu \mathrm{g} / \mathrm{mL}$} & \multicolumn{2}{|c|}{$62.5 \mu \mathrm{g} / \mathrm{mL}$} & \multicolumn{2}{|c|}{$15 \mu \mathrm{g} / \mathrm{mL}$} & \multicolumn{2}{|c|}{$3.75 \mu \mathrm{g} / \mathrm{mL}$} & \multicolumn{2}{|c|}{$1 \mu \mathrm{g} / \mathrm{mL}$} & \multicolumn{2}{|c|}{$0.25 \mu \mathrm{g} / \mathrm{mL}$} \\
\hline & RGR/\% & $\begin{array}{l}\text { 细胞毒 } \\
\text { 性等级 }\end{array}$ & RGR/\% & $\begin{array}{l}\text { 细胞毒 } \\
\text { 性等级 }\end{array}$ & RGR/\% & $\begin{array}{l}\text { 细胞毒 } \\
\text { 性等级 }\end{array}$ & RGR/\% & $\begin{array}{l}\text { 细胞毒 } \\
\text { 性等级 }\end{array}$ & RGR/\% & $\begin{array}{l}\text { 细胞毒 } \\
\text { 性等级 }\end{array}$ & RGR/\% & $\begin{array}{l}\text { 细胞毒 } \\
\text { 性等级 }\end{array}$ \\
\hline HQ & 30.30 & 3 & 35.43 & 3 & 39.28 & 3 & 45.34 & 3 & 47.51 & 3 & 56.17 & 2 \\
\hline 1 & 31.41 & 3 & 34.37 & 3 & 44.60 & 3 & 48.26 & 3 & 51.40 & 2 & 72.85 & 2 \\
\hline $3 a$ & 22.44 & 3 & 29.77 & 3 & 32.52 & 3 & 39.37 & 3 & 42.63 & 3 & 50.07 & 2 \\
\hline $6 a$ & 57.27 & 2 & 61.79 & 2 & 72.65 & 2 & 77.26 & 1 & 82.63 & 1 & & 0 \\
\hline $6 b$ & 62.26 & 2 & 71.20 & 2 & 86.28 & 1 & 94.28 & 1 & & 0 & & 0 \\
\hline $6 c$ & 66.52 & 2 & 72.68 & 2 & 74.49 & 1 & 79.21 & 1 & 84.23 & 1 & & 0 \\
\hline 6d & 54.31 & 2 & 66.27 & 2 & 75.33 & 1 & 81.26 & 1 & 88.23 & 1 & & 0 \\
\hline $6 e$ & 54.33 & 2 & 58.84 & 2 & 62.13 & 2 & 74.83 & 1 & 78.15 & 1 & 72.81 & 1 \\
\hline $6 f$ & 60.46 & 2 & 67.69 & 2 & 76.41 & 1 & 79.32 & 1 & 78.86 & 1 & & 0 \\
\hline $6 \mathrm{~g}$ & 54.49 & 2 & 57.50 & 2 & 68.38 & 2 & 76.47 & 1 & 73.33 & 1 & & 0 \\
\hline $6 \mathrm{~h}$ & 69.45 & 2 & 68.83 & 2 & 78.24 & 1 & 80.94 & 1 & 82.75 & 1 & 86.30 & 1 \\
\hline
\end{tabular}

表 3 目标化合物光毒性的测定结果 ${ }^{a}$

Table 3 The results of target compounds of phototoxicity determination

\begin{tabular}{cccccccccccc}
\hline 化合物 & $\mathrm{HQ}$ & $\mathbf{1}$ & $\mathbf{3 a}$ & $\mathbf{6 a}$ & $\mathbf{6 b}$ & $\mathbf{6 c}$ & $\mathbf{6 d}$ & $\mathbf{6 e}$ & $\mathbf{6 f}$ & $\mathbf{6 g}$ & $\mathbf{6 h}$ \\
\hline PIF 值 & 27.782 & 11.054 & 9.385 & $* 1.0$ & 1.331 & $* 1.0$ & $* 1.0$ & 1.385 & 1.067 & 2.674 & $* 1.0$ \\
\hline
\end{tabular}

a受试物在最高受试浓度仍然没有显示任何细胞毒性(阳性和阴性的读数均成直线), 用 “PIF $=* 1.0 ”$ 表示无潜在光毒性.

致谢 本工作中的酪氨酸酶活性抑制及其毒性和光毒 性实验由四川省化妆品工程技术研究中心实验室完成, 所有谱图和相关数据由四川大学分析测试中心提供, 在 此表示衷心的感谢.

\section{References}

[1] Soliman, K.; Ohad, N.; Ramadam, M.; Snait, T.; Tal, P.; Jacob, V. J. Med. Chem. 2007, 50, 2676.

[2] Sabrina, O.; Delphine, R.; Sebastien, B.; Anne, M. M.; Eric, P.; Ahcene, B. J. Med. Chem. 2006, 49, 329.

[3] Kim, Y. J.; Joo, E. C.; Motoichi, K.; Hiroshi, V.; Shiro, K. Biomacromolecules 2004, 5, 474.

[4] Zhang, C.-X.; Yin, H.-P. Chin. J. Biochem. Pharm. 2005, 26, 2 (in Chinese).

(张春香, 尹鸿萍，中国生物药物杂志, 2005, 26, 2.)

[5] Rainer, K. U.; Martin, A. P. Carbohydr. Res. 2000, 325, 72.

[6] Ernest, V. C.; Cecil, K.; Heino, H.; Hansruedi, G. Biochem. Pharmacol. 1999, 57, 663.

[7] Li, K.-L.; Chen, L.-Z.; Liu, W.-Z.; Lei, G.-L.; Zhang, Y.-Z. China
Pharmacist 1998, 1, 1 (in Chinese).

(李开兰, 陈立中, 刘文芝, 雷观鲁, 张谊芝, 中国药师, 1998, 1, 1.)

[8] Yang, Y.-W.; Zhu, Y. J. Environ. Health 2010, 27, 8 (in Chinese). (杨艳伟, 朱英, 环境与健康杂志, 2010, 27, 8.)

[9] Stephen, H.; Oscar, M. S.; Vincent, M.; Wolfgang, M. Tetrahedron. 2001, $57,3267$.

[10] (a) Hamid, R.; Rotshteyn, Y. Toxicol. In Vitro 2004, $18,5$. (b) Zheng, Y.-T.; Bi, K.-L. J. Immunol. 1992, 8, 226 (in Chinese). (郑永唐, 贲昆龙, 免疫学杂志, 1992, 8, 266.)

[11] (a) Ministry of Health of the People's Republic of China Hygienic Standard Cosmetics 2007, p. 115 (in Chinese).

(中华人民共和国卫生部, 化妆品卫生规范, 2007, p. 115.)

(b) EC EU67/548/EEC Appendix V B. 41 In Vitro 3T3 NRU phototoxicity test OECD guideline for testing of chemicals, 2000.

[12] Yan, Y.; Qin, B.; Ren, C.-L.; Chen, X.-Y.; Yip, Y. K.; Ye, R. J.; Zhang, D. W.; Su, H. B.; Zeng, H.-Q. J. Am. Chem. Soc. 2010, 132, 5869.

[13] Li, Q.-R.; Gu, C.-Z.; Yin, H.; Zhang, Y. Chin. J. Org. Chem. 2005, 25, 11 (in Chinese).

(李前荣, 顾承志, 尹浩, 张毅, 有机化学, 2005, 25, 11.)

(Zhao, C.) 